\title{
A Cellular Automaton Model for the Keep-Right-Except-To-Pass Rule
}

\author{
Yi ZHANG, Yifan ZHANG, Yu GUO, Weihai LI, Qi WANG \\ School of Electronic Engineering, Beijing University of Posts and Telecommunications, Beiing 100876,China
}

\begin{abstract}
This paper aims at examining the effectiveness of the Keep-Right-Except-To-Pass rule in different traffic conditions. In addition, the paper tries to explore a more rational traffic rule to reach a better balance between traffic flow and safety. To address the problem, we develop a two-lane cellular automaton model. In our model, we divide the vehicles into two categories according to their willingness to overtake and emphasize on the overtaking behavior of the vehicles based on the Keep-Right-Except-To-Pass rule.

To test the rule, we check the relation between traffic density and traffic flow. And we find adopting the rule can raise the traffic flow by an average of $21.4 \%$ in light traffic and $24.8 \%$ in high traffic compared to not allowing overtaking. This means this rule of overtaking can improve the efficiency of traffic. Moreover, we develop our own Convert-Only-Once rule on the basis of our model. And simulation proves that compared to the Keep-Right-Except-To-Pass rule, our new rule can boost the traffic flow by $14.3 \%$ and enhance the safety index $\mathrm{K}$ by $1.2 \%$.

Furthermore, we analyze the influence of the Coriolis force on our model when we adjust our model to the situation of driving on the left. In order to fit our model into an intelligent system, we exclude the influence of willingness to overtake and make some adjustment to a certain parameters. And the conclusion is similar, Convert-Only-Once rule can still hold $8.4 \%$ more traffic flow than the Keep-Right-Except-To-Pass rule.

KEYWORD: Traffic rule; Cellular automaton model; Traffic flow
\end{abstract}

\section{INTRODUCTION}

\subsection{Introduction}

In contemporary society, setting up a traffic rule to balance between the overtaking and keeping-safedistance is indeed a significant issue. And a rational traffic rule should reach the tradeoff between the traffic flow and safety. Thus, two main purposes of our model are 1) examining the Keep-Right-ExceptTo-Pass rule, 2) adjusting the rules to traffic flow in practice.

\subsection{Nomenclatures}

The Table1 is used to explain the meaning of the variables in the formula.

\subsection{Assumption and Simplification}

We only study the traffic flow on the straight lane without entrance or exit. We neglect the process of entering and leaving the lanes of vehicles but only care about the traffic flow of a specific length of freeway.

One of the lanes is used for traveling only and the other lane is used for overtaking only.

The traffic flow of both lanes is in the same direction. Thus we exclude the situation of driving in the adverse direction.

The physical appearance and the practical performance of the vehicles are identical. In order to simplify the model, we determine the vehicles are absolutely the same in terms of type, size and the function.

We ignore the driving proficiency of the drivers. Thus the conditions of vehicles are determined by the drivers' strategies.

We simply put the vehicles' strategies into two categories: the adventurous and the conservative. Although the drivers' physical and psychological factors play a part in decision-making (to overtake or not), we simply focus on the effect of vehicles' strategies in our model. 
Table 1. Nomenclatures

\begin{tabular}{|c|c|}
\hline Lane 1 & The overtaking lane \\
\hline Lane 2 & The travel lane \\
\hline$i$ & The serial number of the vehicle \\
\hline$t$ & Moment of time \\
\hline$x_{i}(t)$ & $\begin{array}{l}\text { The position of the } \text { vehicle }_{i} \text { at the moment } t \\
\text { on Lane } 2\end{array}$ \\
\hline $\operatorname{gap}_{i}(t)$ & $\begin{array}{l}\text { The number of the cells between the } \text { vehicle }_{i} \\
\text { and } \text { vehicle }_{i+1} \text { on Lane } 2\end{array}$ \\
\hline$g a p_{i}^{\prime}(\mathrm{t})$ & $\begin{array}{l}\text { The number of the cells between the } \text { vehicle }_{i} \\
\text { and vehicle } e_{i+1} \text { on Lane } 1\end{array}$ \\
\hline$v_{i}(t)$ & $\begin{array}{l}\text { The velocity of the } \text { vehicle }_{i} \text { at the moment } t \\
\text { on the Lane } 2\end{array}$ \\
\hline$v_{i}^{\prime}(t)$ & $\begin{array}{l}\text { The velocity of the } \text { vehicle }_{i} \text { at the moment } t \text { on } \\
\text { the Lane } 1\end{array}$ \\
\hline$P_{A}$ & $\begin{array}{l}\text { The overtaking probability of the adventurous } \\
\text { drivers }\end{array}$ \\
\hline$P_{C}$ & $\begin{array}{l}\text { The overtaking probability of the conservative } \\
\text { drivers. }\end{array}$ \\
\hline$\rho(t)$ & The density of vehicles \\
\hline$N(t)$ & $\begin{array}{l}\text { The quantity of the vehicles on our two-lane } \\
\text { freeway. }\end{array}$ \\
\hline $\bar{v}(\mathrm{t})$ & Average velocity. \\
\hline$L$ & The length of each lane \\
\hline$j$ & The serial number of the lane \\
\hline$J(t)$ & The amount of traffic flow \\
\hline K & The safety at a particular moment \\
\hline$F_{A}$ & The proportion of adventurous vehicle \\
\hline $\mathrm{F}$ & Coriolis force \\
\hline $\mathrm{F}$ & The friction that ground can provide \\
\hline
\end{tabular}

\section{SETTING UP THE MODEL}

\subsection{Preparing the new file with the correct template}

We model the physical structure of the freeways as a two-lane cellular automaton. The length of each lane is $\mathrm{L}$, that is, the number of the cells in the array is $\mathrm{L}$. And each cell can be designated as a vehicle or a vacancy. The model depends on the track of the individual vehicles running on the freeway and the distance between the two vehicles can be calculated by counting the number of the vacant cells. Particularly, the distance between vehicle $_{i}$ and is vehicle $_{i+1}$

$$
\operatorname{gap}_{i}(t)=x_{i+1}(t)-x_{i}(\mathrm{t})-1
$$

We take as the time interval for the automaton to change from one condition to its next condition. We focus on the vehicles in the automaton, whose condition at one moment of time, including position and velocity, are decided by the former moment. Velocity of vehicles can be

$$
v_{i}(\mathrm{t})=\left\{0,1,2,3 \ldots \ldots \mathrm{v}_{\max }\right\}
$$

which represents the number of cells it can pass through in one time interval. And v_max represents the velocity limitation on the lane. In our following explanation, we'll define the deduction rules for vehicles on different lanes, with which we can deduce a vehicle's next condition with its current one.

We classify the vehicles' behavior on the lane as two types. One is Following Behavior, which is, running on the travel lane without overtaking. The other is Overtaking Behavior, which means one vehicle overtakes the front vehicle and then returns to the travel lane. At any moment, a vehicle follows the front or changes lanes to overtake.

Then, we will illustrate the deduction rules of Following Behavior on the travel lane and of Overtaking Behavior respectively.

\subsection{The deductive rule of following behavior}

Based on previous simplification, we classify the drivers' strategies as adventurous ones and conservative ones. The two types of vehicles' behavior have distinct deductive rules in Following Behavior

\section{About the conservative strategy}

The conservative ones are inclined to keep the safe distance. They slow down when they get close to the front car to avoid collision. We assume vehicle $_{i}$ takes conservative strategy. We set up principles for deduction to simulate the process of vehicle $_{i}$ following vehicle $e_{i+1}$.on the Lane 2 .

Step 1: Acceleration:

When $v_{i}(t)<$ gap $_{i}$, vehicle ${ }_{i}$ will accelerate to narrow the gap, and the velocity of this progress is

$$
v_{i} \rightarrow v_{i(t)}=\min \left(v_{i}(t)+1, v_{\max }\right)
$$

Step 2: Affirmative deceleration (In case of running into the front car)

When $v_{i}(\mathrm{t})>\operatorname{gap}_{i}(t)$, will decelerate in order to keep the safe distance. The velocity after this process is

$$
v_{i}\left(t+\frac{1}{3}\right) \rightarrow v_{i}\left(t+\frac{2}{3}\right)=\min \left(\operatorname{gap}_{i}(t), v_{i}\left(t+\frac{2}{3}\right)\right)
$$

Step3: Random deceleration

According to the justification of the front car and its own, vehicle $e_{i}$ will random decelerate. The velocity of random deceleration is:

$$
\begin{aligned}
& v_{i}\left(t+\frac{2}{3}\right) \rightarrow v_{i}(t+1) \\
& =\left\{\begin{array}{l}
\max \left(v_{i}\left(t+\frac{2}{3}\right), 0\right) \text { the probability is } p_{c o n} \\
v_{i}\left(t+\frac{2}{3}\right)
\end{array}\right.
\end{aligned}
$$

Then we can work out the changing position of the vehicle ${ }_{i}$ by calculating the number of the cells.

$$
x_{i}(t) \rightarrow x_{i}(t+1)=x_{i}(t)+v_{i}(t+1)
$$




\section{About the adventurous strategy}

The adventurous strategy tends to speed up. They would keep the maximum velocity and keep a shorter distance with the front car. We assume vehicle $_{i}$ takes adventurous strategy and then simulate the progress of the vehicle $_{i}$ following vehicle vit1 . Similar to conservative strategy, we define the process into 2 steps.

Step1: Acceleration:

$v_{i}(t) \rightarrow v_{i}\left(t+\frac{1}{2}\right)=\min \left(\operatorname{gap}_{i}(t), v_{\max }\right)$

Step2: Random deceleration

$$
\begin{aligned}
v_{i}\left(t+\frac{1}{2}\right) \rightarrow v_{i}(t+1) & \left\{\begin{array}{l}
\max \left(v_{i}\left(t+\frac{1}{2}\right), 0\right) \text { the probability is } p_{A D} \\
v_{i}\left(t+\frac{1}{2}\right)
\end{array}\right.
\end{aligned}
$$

Changing position of the vehicle $_{i}$

$$
x_{i}(t) \rightarrow x_{i}(t+1)=x_{i}(t)+v_{i}(t+1)
$$

\subsection{The deductive rules of overtaking}

We set up two sub-progress of the overtaking

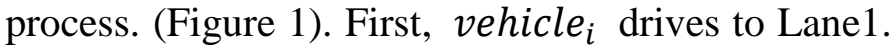
In the next time moment, when vehicle $_{i}$ overtakes vehicle $_{i+1}$.and drives back to Lane 2 . Thus the process of overtaking consists of two consecutive moments of time and the vehicles can't drive on Lane 1 for more than one time interval.
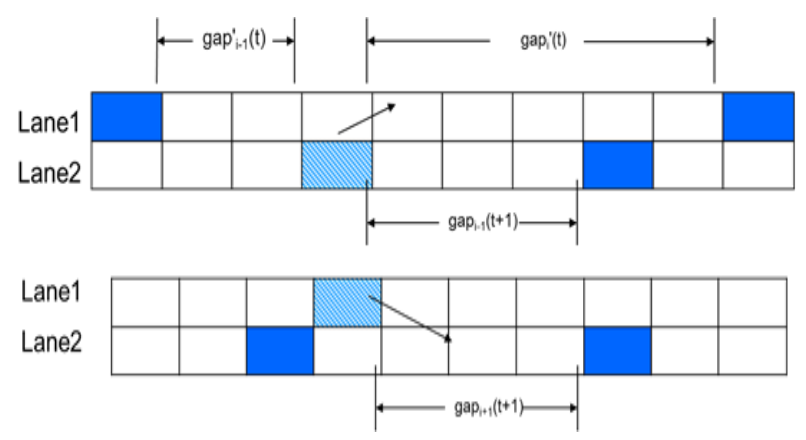

Fig. 1 The deductive rules of overtaking

In what conditions can an individual vehicle finish an overtaking? Our model sets two requirements to decide whether the vehicle can make a successful overtaking. The first requirement is Requirement for Velocity. If the velocity of vehicle $_{i}$ meets the condition that $v_{i}(t)<g a p_{i}$, it is impossible for vehicle $_{i}$ to finish overtaking in the next time interval $t+1$. So it will just follow vehicle vit1 . We can define Requirement for Velocity as $v_{i}(t)>$ gap $_{i}$

After meeting the requirement above, we must take safety into consideration. We define the other requirement for overtaking, the Requirement for
Safety. When this requirement cannot be satisfied, the vehicle should follow the front to avoid collision. As shown in Figure 1, there must be enough space on Lane 1 for vehicle $_{i}$ to overtake. What's more, the following vehicle $i_{-1}^{\prime}$ on the Lane 1 cannot collide into the vehicle ${ }_{i}$. So the Requirement for Safety can be denoted as

$$
\operatorname{gap}_{i}{ }^{\prime}(t)>\operatorname{gap}_{i}(t) \& \operatorname{gap}_{i-1}^{\prime}(t) \geq \min \left(v_{i-1}^{\prime}(t)+1, v_{\text {max }}^{\prime}\right)(10)
$$

In the expressions above, the definitions of $\operatorname{gap}_{i}{ }^{\prime}(t)$ and gap $_{i-1}^{\prime}(t)$ are as follows:

$$
\begin{aligned}
& \operatorname{gap}_{i}(t)=x_{i+1}^{\prime}(t)-x_{i}(t)-1 \\
& \operatorname{gap}_{i-1}^{\prime}(t)=x_{i}(t)-x_{i-1}(t)-1
\end{aligned}
$$

We can figure out the safe velocity when vehicle $_{i}$ choose to overtake front vehicle

$$
v^{\prime}(t+1)=\min \left\{v_{i}(t), \operatorname{gap}_{i}{ }_{i}(t), v_{\max }\right\}
$$

Once the vehicle passes through the front car on Lane 1, it must return to Lane 2 on the next time moment. To determine the range of the velocity when vehicle $_{i}$ return to the Lane 2, we should ensure vehicle $_{i}$ cannot be collided by vehicle $e_{i-1}$ and would not run into vehicle vit1 $_{1}$. (described in Figure 1)We can figure out the safe velocity when vehicle $_{i}$ chooses to return to the Lane 2.

$$
v_{i}(t+2)=\max \left(\operatorname{gap}_{i+1}(t+1), v_{i}(t+1)\right)
$$

Overall, we can figure out the position change of vehicle $_{i}$ during a complete process of overtaking.

$$
\left\{\begin{array}{c}
x_{i}^{\prime}(t+1)=x_{i}(t)+v_{i}^{\prime}(t+1) \\
x_{i}(t+2)=x_{i}^{\prime}(t+1)+v_{i}(t+2)
\end{array}\right.
$$

What's more, to distinguish two types of strategies, we define the concept of "preference rate" in our model. This rate determines the preference for overtaking when either two of the strategies meets the two requirements. Based on our simplification, the preference rate of adventurous strategy is higher than that of the conservative one, that is, $P_{A}>P_{C}$. To be more precise, we regard the preference rate as the probability of choosing to pass through when they meet the requirement to overtake. The value of $P_{A}$ and $P_{C}$ are determined by life experience, but to make it convenient for us to test our model, we set the values as $P_{A}=0.9, P_{C}=0.5$.

\subsection{Index to test the model}

To test our model, we set up several indexes as the standard.

$F_{A}$ : The proportion of vehicles taking the adventurous strategy.

$\rho(\mathrm{t})=\frac{N(t)}{2 L}$ We define whether the traffic is heavy or light by adopting the density of vehicles. 
We define $\rho \in(0.1,0.3)$ as light traffic and $\rho \in(0.6,0.8)$ as heavy traffic.

$$
\bar{v}(\mathrm{t})=\frac{1}{N(t)} \sum_{j=1}^{2} \sum_{i-1}^{N_{j}(t)} v_{(j, i)}(t)
$$

We acknowledge that the safety level on the lane is affected by the average speed of vehicles. In common sense, the larger $\overline{v(t)}$ is, the lower safety level the lane has.

$\mathrm{J}(\mathrm{t})=\rho(\mathrm{t}) * \overline{v(t)}$ We measure the traffic flow by using the index of $J(t)$, which means the number of the vehicles on the two lanes at the moment $t$. Larger $J(t)$ means higher traffic flow.

$$
f_{s}(t)=\frac{N_{S}(t)}{N(t)} \text { We adopt the index } f_{s}(t) \text { to }
$$

examine the ratio of vehicles making overtaking process at moment $t$. The safety level is also influenced by this index because accidents are more likely to happen when vehicles overtake.

$$
\mathrm{K}=\frac{1}{f_{s} * \overline{v(t)}+1} \text { We define the safety coefficient } \mathrm{K}
$$
with an empirical formula.

\subsection{Results and analysis}

We run the simulation where our lanes are made of 1000 cells, that is, $L=1000$ and we set 30000 steps for our simulation. Then, we analyze through the last 20000 steps whose the data is stable. Under the conditions of $v_{\max }=5, \quad P_{A D}=P_{C O N}=0.5$, $P_{A}=0.8, P_{C}=0.5$. We can get the relationship of the density of vehicle and the traffic flow. We exhibit the results in Figure 2.

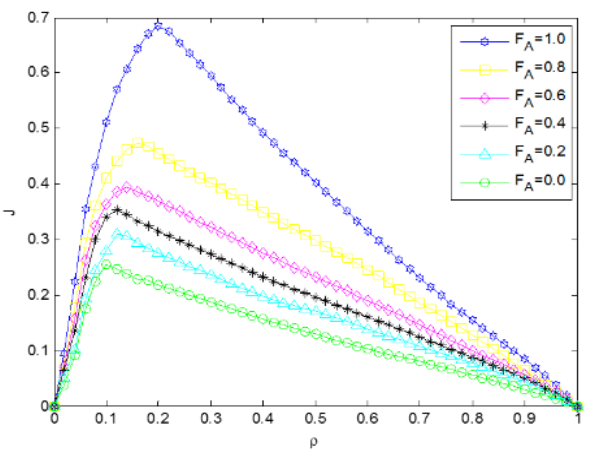

Fig.2 The X-axis represents the density of vehicles, and the Yaxis represents the traffic flow $\mathrm{J}$.

From the Figure 2, we can see that when $\rho \in(0.1,0.3)$, the traffic flow is higher, and when $\rho \in(0.6,0.8)$, the traffic flow is lower. Thus, we can get to the conclusion that it is better to increase the traffic flow under the light traffic. Meanwhile, the traffic flow is related with proportion of the drivers' strategies (adventurous or conservative) under the same density of the density of vehicles.

In the following analysis, we examine the relationship between the density of vehicles $\rho$ and the safety coefficient K. We show the result in Figure3.

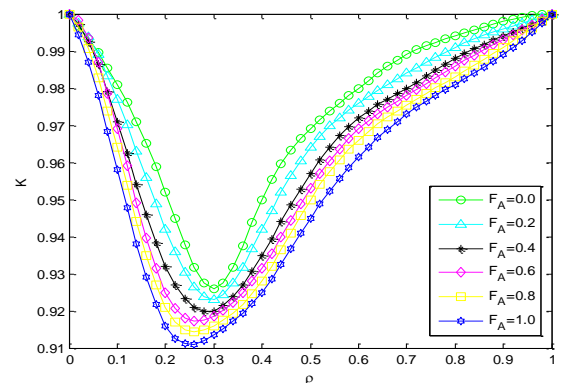

Fig. 3 The $\mathrm{X}$-axis represents the density of the vehicles, the $\mathrm{Y}$ axis represents the coefficient of safety.

To check the effectiveness of the Keep-RightExcept-To-Pass rule, we change the parameters $P_{A}=P_{C}=0$. This means that all the vehicles are not willing to overtake the front vehicle and each vehicle in our model simply follows the front vehicle. According to our definition of safety, $K=1$. We exhibit the relationship between $J$ and $\rho$ by Figure 4 . We take $F_{A}=0.4$ and $F_{A}=0.8$ as the example to illustrate.

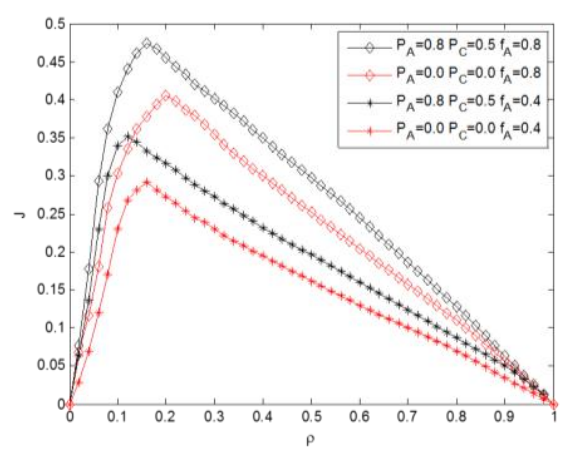

Fig.4 the relationship between $\mathrm{J}$ and $\rho$

From the Figure above, we can easily conclude that the quantities of traffic flow increase under the circumstance of identical density and proportion of the aggressive when we adopt the Keep-RightExcept-To-Pass-Rule. Thus, we justify that the traffic rules of overtaking can increase the quantities of the traffic flow to certain extent. In fact, according to our calculation, the average increasing rate is $21.4 \%$ in low traffic and $24.8 \%$ in high traffic.

Then we change the $v_{\max }$ under the condition of $F_{A}=0.4$. to examine the effect of speed limit on our model. We get the curve in Figure 5 to demonstrate the influence between traffic flow and coefficient of safety. With the increase of $v_{\max }$, the traffic flow raise significantly and there's also a drop in the safety coefficient.
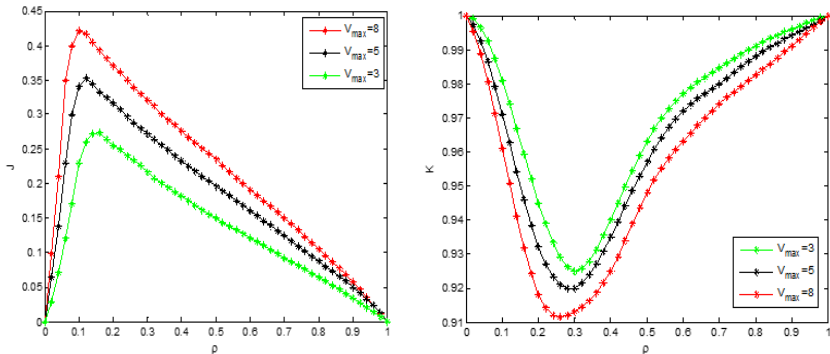

Fig.5 influence between traffic flow and coefficient of safety 


\subsection{A better traffic rule}

In the previous analysis, we have already proved that the adoption of Keep-Right-Except-To-Pass rule can raise traffic flow in comparison with not allowing overtaking. Now we are going to explore a better rule to ensure a larger traffic flow both in light and heavy traffic without significantly affecting safety coefficient $\mathrm{K}$.

Consider our definition of "the deductive rule of overtaking". We make some adjustment to the overtaking rules to form our own Convert-OnlyOnce rule:

(1)When one vehicle converts to Lane 1 to pass through the front one, it will continue to drive on the Lane 1;

(2)Driving on the Lane 1 for a long period is permitted, and the vehicles on Lane 1 obey the same rule of Following Behavior as that on Lane 2;

(3)Vehicles on Lane 1 are not allowed to take Lane 2 to complete its overtaking process, that is, overtaking is not allowed on Lane 1 but only allowed on Lane 2.

The adjustment above can raise the utilization rate of the Lane 1. Meanwhile, the raise in the number of vehicles on the Lane 1 makes it more difficult to satisfy the requirements of overtaking at certain traffic density, which means greater safety.

The adjustment above can raise the utilization rate of the Lane 1. Meanwhile, the raise in the number of vehicles on the Lane 1 makes it more difficult to satisfy the requirements of overtaking at certain traffic density, which means higher safety level.

To test our rule, we take $F_{A}=0.4, \mathrm{v}_{\max }=5$ and run the simulation. The distinction in traffic flow and safety coefficient $\mathrm{K}$ between the Keep-RightExcept-To-Pass rules and our Convert-Only-Once rule are presented in Figure 6.
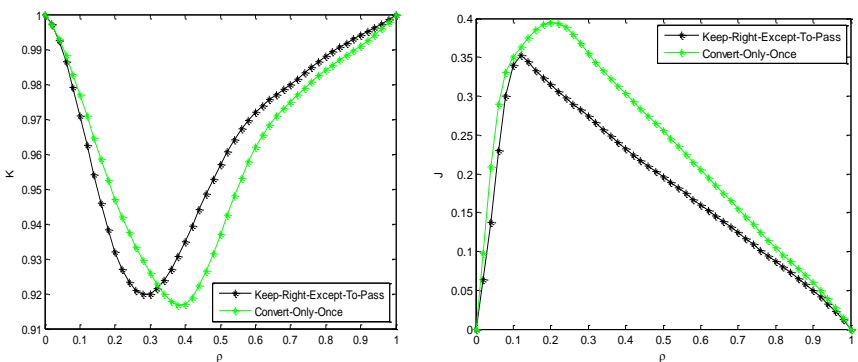

Fig. 6 The distinction in traffic flow and safety coefficient $\mathrm{K}$

We can conclude that in light traffic, our rule ensures greater traffic flow and better safety. As for highway traffic, traffic flow increases but there's a decline on safety. Actually, on average, according to our calculated results, the Convert-Only-Once rule can increase the the traffic flow by $21.4 \%$ in light traffic and $24.8 \%$ in high traffic compared to not allowing overtaking. As for the safety coefficient K, the Convert-Only-Once rule increases $\mathrm{K}$ at about $1.4 \%$ on average in light traffic, but decreases at about $1.2 \%$ on average in heavy traffic. As a whole, our model can significantly raise the traffic flow without exerting much influence on safety level.

\section{FURTHER DISCUSSION}

\subsection{Headings}

According to our previous simulation, the proportion of the adventurous strategy has a positive effect on the traffic flow. What's more, the adoption of overtaking action can also increase the traffic flow. As safety is no longer a big issue, we can set higher speed limitation to encourage larger flow. So we exhibit the adjustment to our model as follows:

- $F_{A}=1$ All the vehicles would take adventurous strategy to ride on the road.

- $P_{A}=1$ All the vehicles must overtake the front ones when they meets the requirements for overtaking.

- $v_{\max }=8$ Raise the velocity limitation of the road in order to enhance the traffic flow.

We can run the simulation of the Keep-RightExcept-To-Pass rule and our Convert-Only-Once rule. The calculation result reveals that there's no major distinction to our previous conclusion. The Convert-Only-Once rule can still promote the traffic flow, but the rate declines to $8.4 \%$.

\subsection{Conclusions}

Based on our cellular automaton model above, we can reach the following conclusions:

(1)The Keep-Right-Except-To-Pass rule is effective in promoting better traffic flow in comparison with not allowing overtaking.

(2)The Convert-Only-Once rule is more rational in boosting the traffic flow. According to our analysis, this rule enhances the utilization rate of Lane 1 without distinctively affecting the safety.

(3)Our model can fit into countries where driving on the left is the norm with adjustment to a certain parameters.

\section{REFERENCES}

[1] Nagel K and Schrekenberg M 1992 J. Phys. I France 2 2221.

[2] Ishibashi Y and Fukui M 1994 J. Phys. Soc. Japan 632882

[3] Wolfram S 1986 Theory and Application of Cellular Automaton (Singapore: World Scientific).

[4] Lei L, Xue Y, Dai S Q 2003 Acta Phys. Sin. 52 2121(in Chinese).

[5] Kuang H, Kong L J, Liu M R 2004 Acta Phys. Sin. 53 2894.

[6] Mou Y, Zhong C W 2005 Cellular Automaton Model of Traffic Flow Based on Safety Driving, Acta Phys. Sin., Vol.54, No.12 (in Chinese).

[7] Peng L J, Kang R, 2009 One-dimensional Celluar Automaton Model of Traffic Flow Considering Drivers' Features, Acta Phys. Sin. Vol.58, No.2 (in Chinese).

[8] Wang X J 2008 Review of the Process and Dynamics of ITS Research and Developments, Urban Transport of China, Vol.6 No.1 (in Chinese).

[9] Knospe W, Santen L, Schadschneider A, Schreckenberg M 2004 Phys. Rev. E 70016115. 